\title{
Truncation Correction for VOI C-arm CT using Scattered Radiation
}

\author{
Bastian Bier ${ }^{a}$, Andreas Maier ${ }^{a}$, Hannes G. Hofmann ${ }^{a}$, Chris Schwemmer ${ }^{a, b}$, Yan Xia ${ }^{a}$, Tobias \\ Struffert $^{c}$ and Joachim Hornegger ${ }^{a}$ \\ ${ }^{a}$ Pattern Recognition Lab, Department of Computer Science, Friedrich-Alexander-University \\ Erlangen-Nuremberg, Erlangen, Germany; \\ ${ }^{b}$ Erlangen Graduate School in Advanced Optical Technologies (SAOT), \\ Friedrich-Alexander-University Erlangen-Nuremberg, Erlangen, Germany; \\ ${ }^{c}$ Department of Neuroradiology, Universitätsklinikum Erlangen, Germany
}

\begin{abstract}
In C-arm computed tomography, patient dose reduction by volume-of-interest (VOI) imaging is of increasing interest for many clinical applications. A remaining limitation of VOI imaging is the truncation artifact when reconstructing a $3 \mathrm{D}$ volume. It can either be cupping towards the boundaries of the field-of-view (FOV) or an incorrect offset in the Hounsfield values of the reconstructed voxels.

In this paper, we present a new method for correction of truncation artifacts in a collimated scan. When axial or lateral collimation are applied, scattered radiation still reaches the detector and is recorded outside of the FOV. If the full area of the detector is read out we can use this scattered signal to estimate the truncated part of the object. We apply three processing steps: detection of the collimator edge, adjustment of the area outside the FOV, and interpolation of the collimator edge.

Compared to heuristic truncation correction methods we were able to reconstruct high contrast structures like bones outside of the FOV. Inside the FOV we achieved similar reconstruction results as with water cylinder truncation correction. These preliminary results indicate that scattered radiation outside the FOV can be used to improve image quality and further research in this direction seems beneficial.
\end{abstract}

Keywords: Scatter, Truncation, Truncation Correction, C-arm, X-ray, Scatter Estimation, VOI Imaging

\section{INTRODUCTION}

In many applications in neuroradiology or surgery it is required to use intraoperative imaging for guidance of the deployment of small devices like stents or coils. This is often done using a two-dimensional fluoroscopic view on a C-arm angiograph. In order to reduce the radiation exposure for the patient the x-rays are collimated so that only a fraction of the detector size is used for imaging. Sometimes the information of these 2D-images is not sufficient and a $3 \mathrm{D}$ reconstruction of this small FOV is desirable, e.g. for a fluoroscopic overlay ${ }^{1}$.

With common analytical methods like filtered backprojection, the reconstruction result contains artifacts at the boundaries of the FOV. They appear because the algorithm performs a ramp filtering of the projection data. Due to the collimation, an artificial edge appears in the spatial domain of the projection data, by which high frequencies are generated during the filtering step. By backprojecting the filtered image into the volume, these high frequencies generate truncation artifacts ${ }^{2}$.

Due to the collimation, the scan does not contain the full spatial extent of the object. To reconstruct an object exactly, the extent of the object has to be known from a prior $\operatorname{scan}^{3}$, or a small part of the object has to be known ${ }^{4}$. If no prior knowledge is available, heuristic methods have to be applied. These methods are often

Further author information:

B.B.: E-mail: bastian.bier@medtech.stud.uni-erlangen.de

A.M.: E-mail: andreas.maier@cs.fau.de

C.S.: E-mail: chris.schwemmer@cs.fau.de 


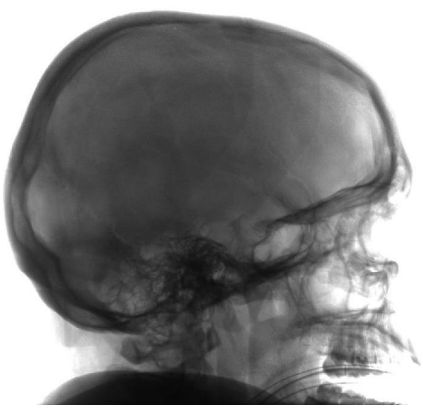

(a) Full projection, grayscale window $(400,2000)$.

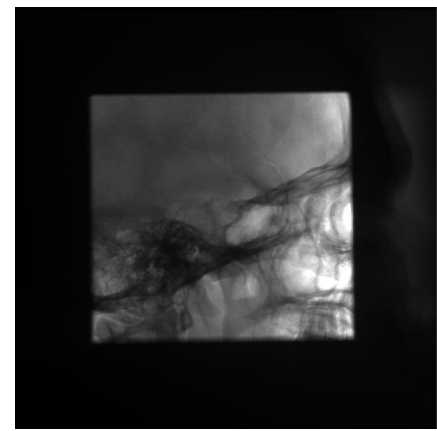

(b) Volume-of-interest projection, grayscale window $(400$, 2000).

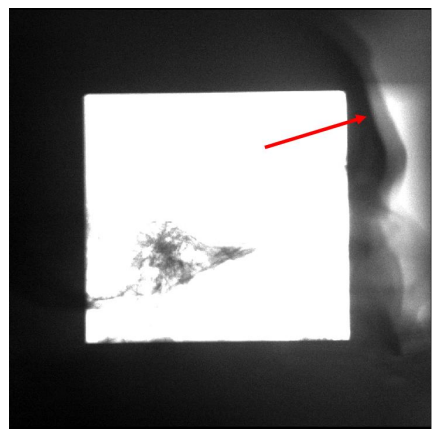

(c) Volume-of-interest projection, grayscale window $(400$, 620 ).

Figure 1: Projection images of the skull of one patient. (1a) full projection. (1b) collimated projection with the same grayscale window. (1c) collimated projection with narrower grayscale window to show the attenuated signal in the outer field. The shape of the head is still visible (marked by the arrow).

called truncation correction in the literature. The better the heuristic fits to the truncated object, the better the resulting reconstruction is. Some methods model the outside of the FOV which has not been measured ${ }^{5,6}$. Others require extrapolation only implicitly ${ }^{7,8}$. With respect to image quality, these heuristic methods provide acceptable results. Chityala et al. present an approach that uses a filter to attenuate the radiation outside the FOV to a minimal dose. In a postprocessing step, this information is then used to solve the truncation problem. The approach presented here is similar to Chityala's paper as we also used a small amount of radiation outside the regular FOV. In contrast to their paper, no additional dose has to be applied to the patient, as we employ the scattered radiation that is caused by the collimator edges and the object itself. This information is always present in a truncated FOV scenario but usually not measured.

In Figure 1, we present projection images of a skull of the same patient. Inside the FOV, the non-collimated (Figure 1a) and the collimated (Figure 1b) projection are almost identical. Figure 1c shows the same image as Figure 1b, just with a different grayscale window. You can see that the attenuated signal caused by the scattered radiation clearly depicts the shadow of the skull (arrow in Figure 1c). Especially if bones are truncated, the effect is strongly visible. With our method, we can use the information scattered radiation provides to reconstruct dense objects outside of the FOV.

\section{MATERIALS AND METHODS}

In Figure 2, the intensities along a line through a collimated projection are plotted. We now divide the projection into three parts: the FOV (area A), the area outside the FOV (area C), and the shadow of the collimator edge (area B). The information outside the FOV is used in our approach to estimate the truncated part of the imaged object. In Figure 2, one can see a rise of the intensity outside the FOV (denoted by the arrow). This is caused by the scattered radiation and marks the end of the skull in the outer FOV. A simple amplification of the scattered radiation outside the FOV is performed, in order to expand the object as much as possible. The workflow of our method can be divided into three steps: First we have to detect the edges of the collimator. After that the signal of the scattered radiation is amplified. Finally, an interpolation is applied to reconstruct the object behind the collimator edges.

\subsection{Edge Detection}

We detect the collimator edge for each row of the projection image. It is modeled not just as a point, but as two points (cf. lines 1 and 2, or 3 and 4 in Figure 2). As can be seen in Figure 2, the curve between both ends of a collimator edge can be approximated by a sigmoid function. Since the adjustment and the interpolation of the collimator edges is done row-wise in projection domain, we only have to detect the vertical collimator edges. Detection was performed by applying the Hough-Transformation (HT) for detection of straight lines ${ }^{9}$. 


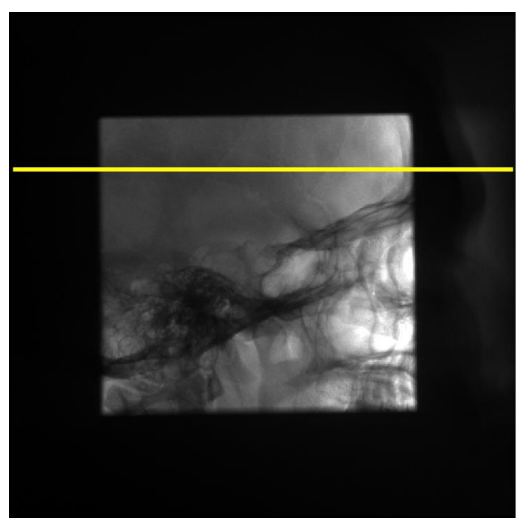

(a) Projection image

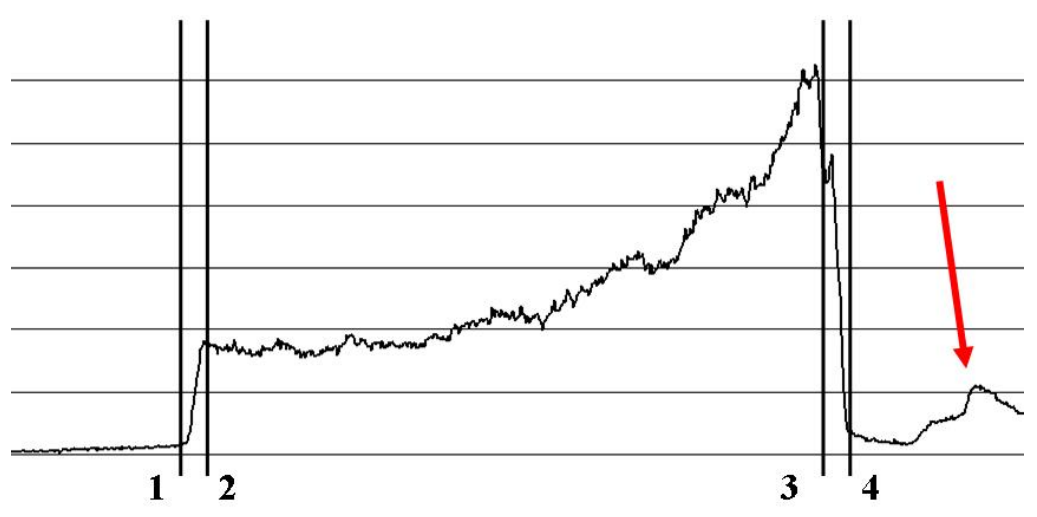

(b) Line plot

Figure 2: The projection is divided into three parts: the FOV (area A), the collimator edge (area B), and the area outside the FOV (area $\mathrm{C}$ ). The lines show where we define the collimator edges. In the edge detection step, the area between the line 1 and 2 , or 3 and 4 are approximated with a sigmoid function.

To reduce processing time we perform the HT only on a region around the collimator position given by the acquisition system. We reduced the search space to an area of \pm 20 pixels around that position. Furthermore, we introduced prior knowledge by the constraint that the edge is nearly vertical in the projection. The result of the HT is the starting point for estimating both ends of the collimator edge. The actual detection is done by fitting a sigmoid function to each line around the region of the given solution of the Hough-Transformation. For increased robustness, we enforced a minimum edge width.

Each edge is now characterized by two points at the top and bottom $5 \%$ signal level of the sigmoid curve, shown in Figure 2. In order to get a continuous edge for the whole projection image, we fit a line through the points by applying the RANSAC algorithm ${ }^{10}$. The four edges are now known for each projection.

\subsection{Adjustment of the outside of the FOV}

With knowledge of the position of the edges, we can adjust (i.e. amplify) the scattered radiation outside the FOV. The adjustment consists of two steps: an amplification and a weighting. For the amplification, we either use an additive or an multiplicative model to bring the area outside the FOV to the same level as the inside. Therefore, we calculate the difference between the first pixel of the outside of the field of view and the outer pixel of the FOV. This difference is then used for the amplification of the outside of the FOV. The second step is the weighting step. The outside of the FOV is either weighted with a linear or a squared function to get a decrease towards the boundary of the FOV. The weighting function is 0 at the boundary of the detector and 1 at the beginning of the FOV for both functions. The result of this adjustment step is seen in Figure $3 \mathrm{~b}$.

\subsection{Interpolation in the area of the Collimator Shadow}

When the outside of the FOV has been adjusted, the last step of the algorithm is performed: the interpolation in the area of the collimator shadow (area B). For the interpolation we applied different methods: Linear interpolation, polynomial interpolation (degree 3), Substract and Shift (SaS) ${ }^{11}$ and a combination of the SaS algorithm with the polynomial interpolation. A resulting line profile after the interpolation step is shown in Figure 3c.

\subsection{Clinical Datasets}

We used clinical datasets for our evaluation. In some cases we got a full projection scan and a truncated scan. This is beneficial for measuring the error, if the reference, the full projection dataset, is given. When the reference reconstruction is given, the correlation coefficient (CC) and the structural similarity (SSIM) of the water cylinder correction and the new approach using scattered radiation is calculated inside the FOV. In one 


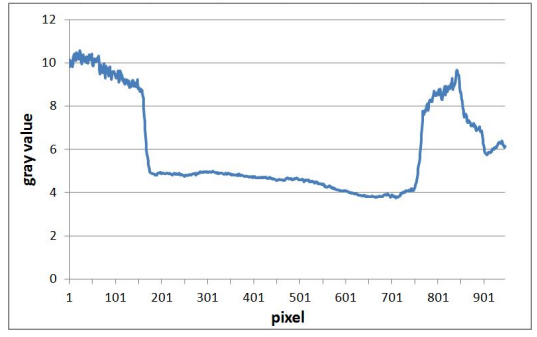

(a) Original line of the projection image in the line integral domain.

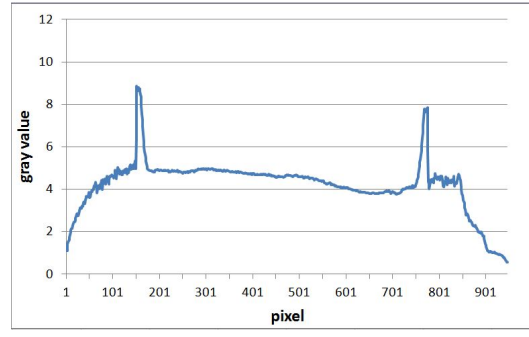

(b) Line profile after the adjustment step. Here, the add+squared method is used.

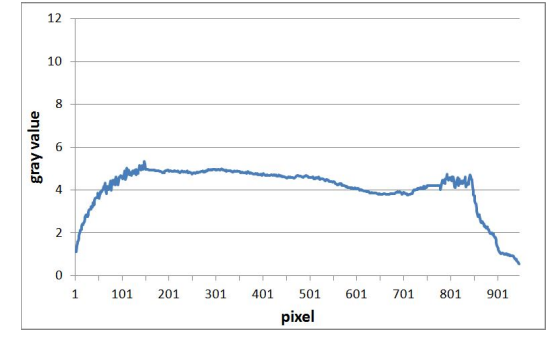

(c) Line profile after the interpolation of the collimator shadow.

Figure 3: Processing steps of a row of a projection.

case only a collimated projection dataset exists. Therefore, evaluation is only performed in a qualitative way for this dataset. All the shown reconstruction results are performed with a volume of $512 \times 512 \times 512$ and a voxel size of $0.4 \mathrm{~mm}$.

For the evaluation of our new approach, the add+squared method for the adjustment step and the linear interpolation is used. Each time a reference data exists, the same slice of the full reference reconstruction, the water cylinder corrected ${ }^{5}$ reconstruction and the reconstruction result of our new approach are shown.

\section{RESULTS}

\subsection{Dataset 1}

The resolution of the projections is $960 \times 960$ with a pixel size of $0.3 \mathrm{~mm}$ and the data consists of 496 projections sampled over $200^{\circ}$ of angulation. The diameter of the VOI is about $9 \mathrm{~cm}$. The dataset was already presented in Chintalapani's paper. A full reference dataset exists and allows the calculation of the error.

In Table 1, one can see the results of the error metrics for the dataset 1 using different combinations of adjustment and interpolation methods. Looking at the CC and the SSIM, the squared weighting results in better values than the linear weighting and the water cylinder corrected results.

In Figure 4, one can see the three different reconstruction results of the same slice: the full reconstruction (Figure 4a), the water cylinder corrected reconstruction (Figure 4b) and our approach using scattered radiation (Figure 4c). In Figure 4c, the bone structure in the reconstruction result outside the FOV (marked by the arrows) is clearly visible. A disadvantage of our method is the drop of intensity in the top left (marked by the ellipse). This drop is located exactly at the boundary of the FOV, in the shadow of the collimator edge, where the interpolation is performed.

In Figure 4d, three line profiles for the different methods are plotted. Here, one can see that the visibility of the bone outside the FOV is improved (see arrow on the left side). Another interesting aspect is the cupping effect of the water cylinder correction. This rise of the intensity towards the outside of the FOV is marked by a second arrow. Such an effect did not appear using our method. Furthermore, the water cylinder correction estimated the object size wrongly compared to the full reconstruction (see circle in Figure 4d). On the right side, both methods did not work properly. Our approach failed, because there was no additional information available far outside the FOV and the water cylinder correction created further cupping.

\subsection{Dataset 2}

The next dataset also shows the skull of a patient. The resolution of the projections is $960 \times 1240$ with a pixel size of $0.3 \mathrm{~mm}$ and the data consists of 496 projections sampled over $200^{\circ}$ of angulation. The diameter of the VOI is about $9 \mathrm{~cm}$. Again, a full reference dataset exists.

In Figure 5, the three different reconstruction results are shown: the reference reconstruction(Figure 5a), the water cylinder reconstruction (Figure 5b) and the new approach with add+squared adjustment and linear interpolation (Figure 5c). Here, the big advantage of the new approach is seen directly: The shape of the 


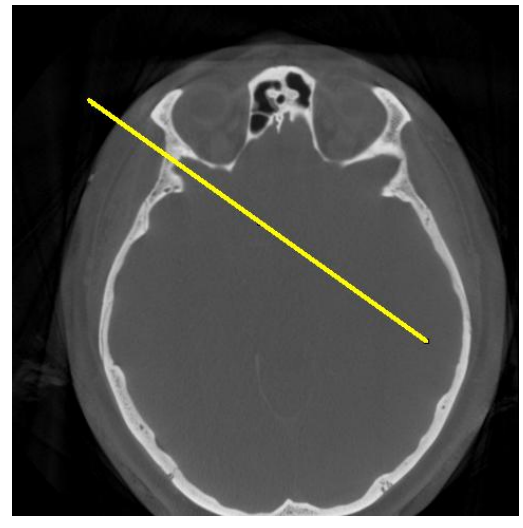

(a) Full scan reconstruction, $\mathrm{HU}$ grayscale window $(-1000,2500)$.

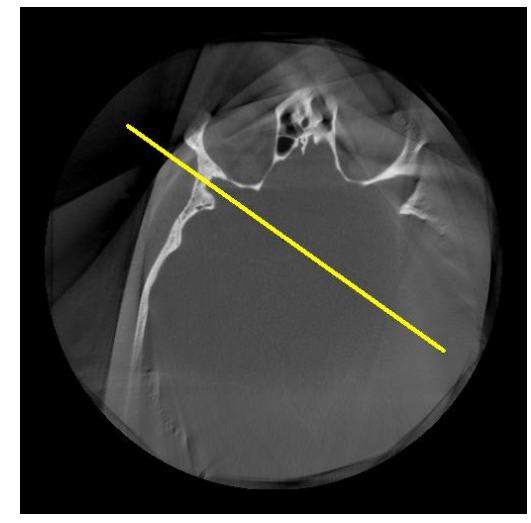

(b) Water cylinder correction, $\mathrm{HU}$ grayscale window $(-1000,2500)$.

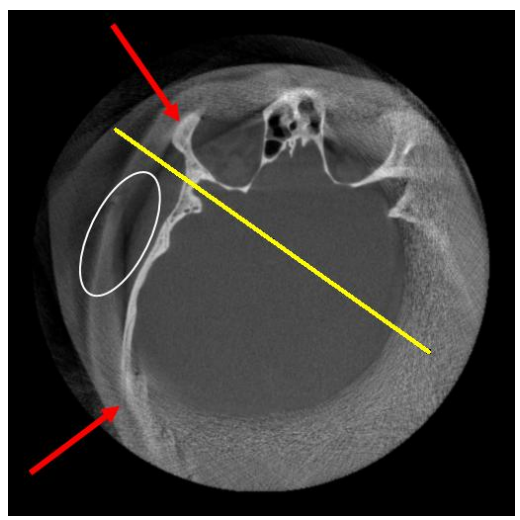

(c) Scattered radiation correction, HU grayscale window $(-1000,2500)$.

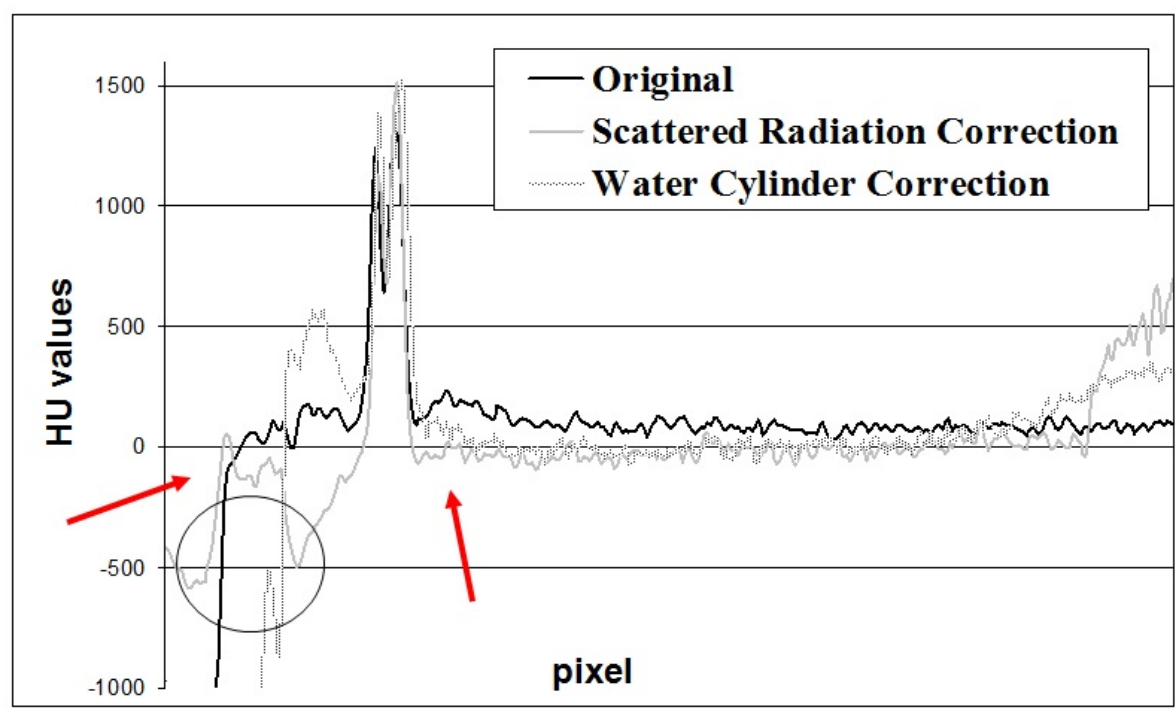

(d) The line profiles of the three different reconstruction methods are shown in this plot.

Figure 4: Results of the full reconstruction, the water cylinder reconstruction and our new approach. The new approach was performed with the add+squared adjustment method and the linear interpolation. Dataset 1.

skull of the patient is clearly visible in the outside of the FOV. In contrast to our new approach, the water cylinder correction shows no further information in the outside of the FOV. The good visual impression of the reconstruction result is reduced by the black circle along the boundary of the FOV, in the area of the collimator shadow. Furthermore, the result of our new approach in this case suffers under an incorrect offset estimation.

The error metrics for the different combination of the methods are shown in Table 2. It shows, that the CC of the new approach is in each case better than the $\mathrm{CC}$ of the water cylinder corrected reconstruction.

\subsection{Dataset 3}

The results of the third dataset are shown in Figure 6. In comparison to the previous datasets, this is a more narrow collimation. The size of this collimation window is about $12 \mathrm{~cm} \times 10 \mathrm{~cm}$. This dataset consists of 496 projections and has a resolution of $960 \times 1240$ with a pixel size of $0.3 \mathrm{~mm}$. A characteristic of this dataset is that motion exists in the projection. This remarks itself in motion artifacts in the reconstruction.

In Table 3, the results of the error measurements are shown. Regarding the correlation coefficient, the new approach is in each case better than the water cylinder correction. 
Table 1: Table of the error metrics for dataset 1.

\begin{tabular}{l|l|c|c}
\hline & & CC & SSIM \\
\hline Water cylinder correction & & 0.9461 & 0.9444 \\
\hline Adjustment Method & Interpolation Method & & \\
\hline Multiply and linear increase & Linear & 0.9371 & 0.9371 \\
& SaS & 0.9373 & 0.9372 \\
& Polynomial Degree 3 & 0.9381 & 0.9380 \\
Multiply and squared increase & Polynomial + SaS & 0.9334 & 0.9334 \\
& Linear & 0.9505 & 0.9452 \\
& SaS & 0.9596 & 0.9453 \\
& Polynomial Degree 3 & 0.9506 & 0.9543 \\
Add and linear increase & Polynomial + SaS & 0.9493 & 0.9449 \\
& Linear & 0.9428 & 0.9426 \\
& SaS & 0.9429 & 0.9427 \\
& Polynomial Degree 3 & 0.9432 & 0.9430 \\
& Polynomial + SaS & 0.9389 & 0.9388 \\
& Linear & 0.9555 & 0.9477 \\
& SaS & 0.9555 & 0.9477 \\
& Polynomial Degree 3 & 0.9555 & 0.9477 \\
& Polynomial + SaS & 0.9550 & 0.9487 \\
\hline
\end{tabular}

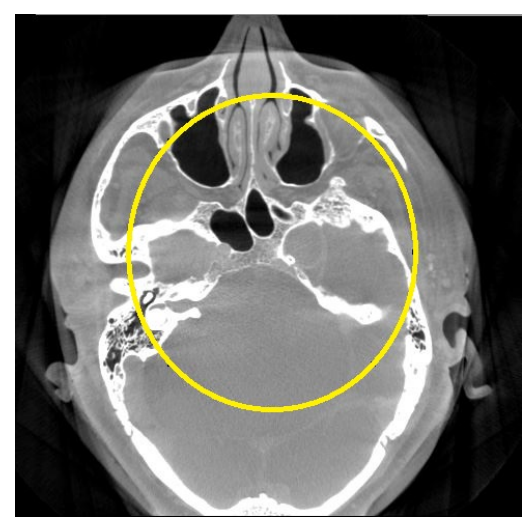

(a) Full scan reconstruction, $\mathrm{HU}$ grayscale window $(-1000,1000)$.

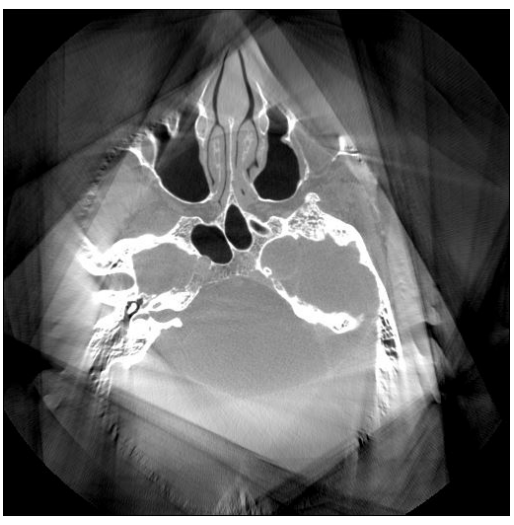

(b) Water cylinder correction, HU grayscale window $(-1000,1000)$.

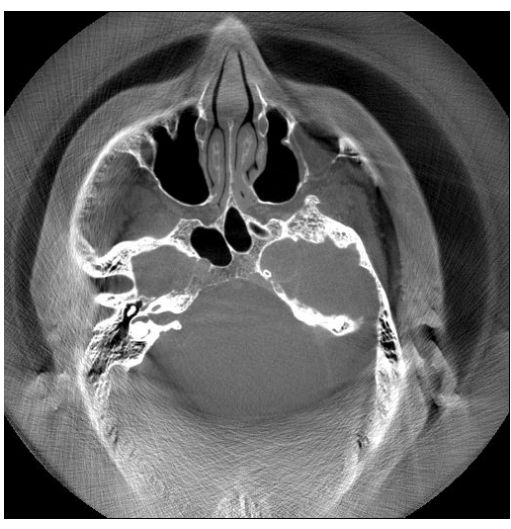

(c) Scattered radiation correction, HU grayscale window $(-1000,1000)$.

Figure 5: Results of the full reconstruction, the water cylinder reconstruction and our new approach. The new approach was performed with the add+squared adjustment method and the linear interpolation. Dataset 2.

In Figure 6c, one can see that the reconstruction result of our new approach contains no usable information in the outside of the FOV. This is caused by less observed scattered radiation outside the FOV due to the smaller collimation size. Despite of that, compared to the water cylinder correction, the error measurements show better results and the offset is more similar to the reference reconstruction. This shows that the new method works also for smaller collimation windows.

\subsection{Dataset 4}

For the following dataset no reference data is available. Hence, the evaluation of the reconstruction result is only done qualitatively. This dataset contains 496 projections and has a pixel size of $0.3 \mathrm{~mm}$. The resolution is $1240 \times 960$. 
Table 2: Table of the error metrics for dataset 2 .

\begin{tabular}{l|l|l|l}
\hline & & CC & SSIM \\
\hline Water cylinder correction & & 0.9228 & 0.9224 \\
\hline Adjustment Method & Interpolation Method & & \\
\hline Multiply and linear increase & Linear & 0.9348 & 0.9262 \\
& SaS & 0.9348 & 0.9261 \\
& Polynomial Degree 3 & 0.9350 & 0.9263 \\
Multiply and squared increase & Polynomial + SaS & 0.9361 & 0.9284 \\
& Linear & 0.9305 & 0.8984 \\
& SaS & 0.9305 & 0.8984 \\
& Polynomial Degree 3 & 0.9308 & 0.8988 \\
Add and linear increase & Polynomial + SaS & 0.9339 & 0.9043 \\
& Linear & 0.9365 & 0.9249 \\
& SaS & 0.9365 & 0.9250 \\
& Polynomial Degree 3 & 0.9364 & 0.9252 \\
& Polynomial + SaS & 0.9383 & 0.9283 \\
Add and squared increase & Linear & 0.9288 & 0.8888 \\
& SaS & 0.9288 & 0.8889 \\
& Polynomial Degree 3 & 0.9295 & 0.8899 \\
& Polynomial + SaS & 0.9339 & 0.8973 \\
\hline
\end{tabular}

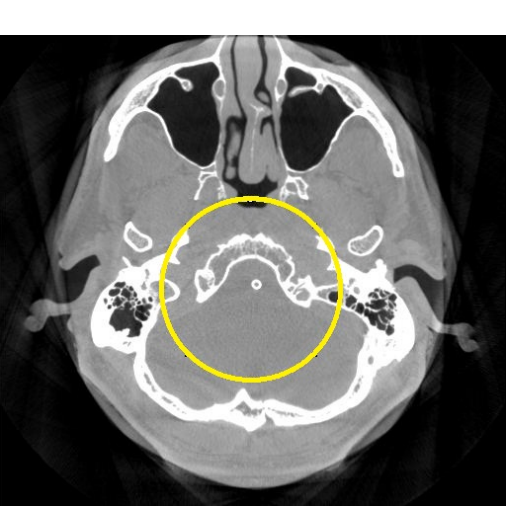

(a) Full scan reconstruction, HU grayscale window $(-1000,1000)$.

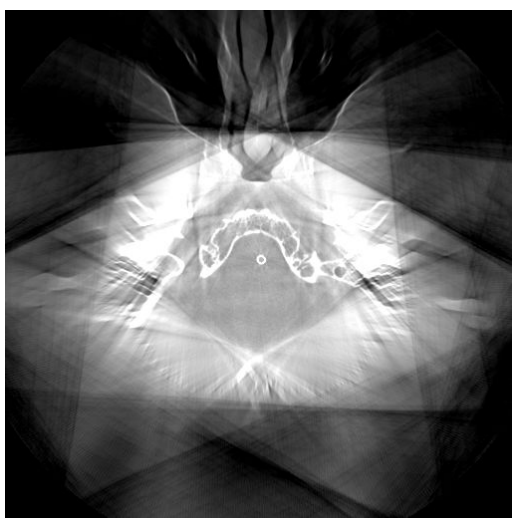

(b) Water cylinder correction, $\mathrm{HU}$ grayscale window $(-1000,1000)$

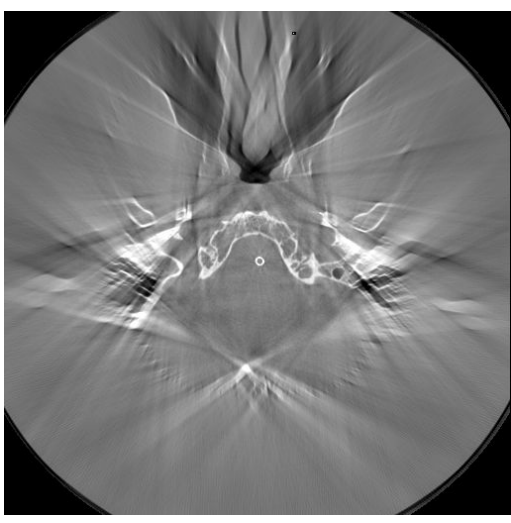

(c) Scattered radiation correction, HU grayscale window $(-1000,1000)$.

Figure 6: Results of the full reconstruction, the water cylinder reconstruction and our new approach. The new approach was performed with the add+squared adjustment method and the linear interpolation. Dataset 3.

In Figure 7, the truncated projection dataset, the water cylinder corrected result and the result of the new approach are shown. The reconstruction of the new approach uses linear+add for adjustment and SaS for interpolation. The big advantage of the new truncation corrected method can be seen again. The estimation of the object outside the FOV clearly shows the contour of the neck. In the water cylinder correction result this cannot be seen. Furthermore, the structures in the FOV look sharper in the image processing approach compared to the water cylinder correction. No cupping-like artifacts can be detected like in the water cylinder correction case. 
Table 3: Table of the error metrics for dataset 3 .

\begin{tabular}{l|l|c|c}
\hline & & CC & SSIM \\
\hline Water cylinder correction & & 0.6907 & 0.67357 \\
\hline Adjustment Method & Interpolation Method & & \\
\hline Multiply and linear increase & Linear & 0.7299 & 0.7037 \\
& SaS & 0.7299 & 0.7037 \\
& Polynomial Degree 3 & 0.7299 & 0.7036 \\
Multiply and squared increase & Polynomial + SaS & 0.7296 & 0.7034 \\
& Linear & 0.7192 & 0.6873 \\
& SaS & 0.7192 & 0.6873 \\
& Polynomial Degree 3 & 0.7192 & 0.6873 \\
Add and linear increase & Polynomial + SaS & 0.7190 & 0.6874 \\
& Linear & 0.7305 & 0.7040 \\
& SaS & 0.7305 & 0.7040 \\
& Polynomial Degree 3 & 0.7305 & 0.7040 \\
& Polynomial + SaS & 0.7302 & 0.7037 \\
Add and squared increase & Linear & 0.7200 & 0.6876 \\
& SaS & 0.7200 & 0.6975 \\
& Polynomial Degree 3 & 0.7201 & 0.6875 \\
& Polynomial + SaS & 0.7199 & 0.6876 \\
\hline
\end{tabular}

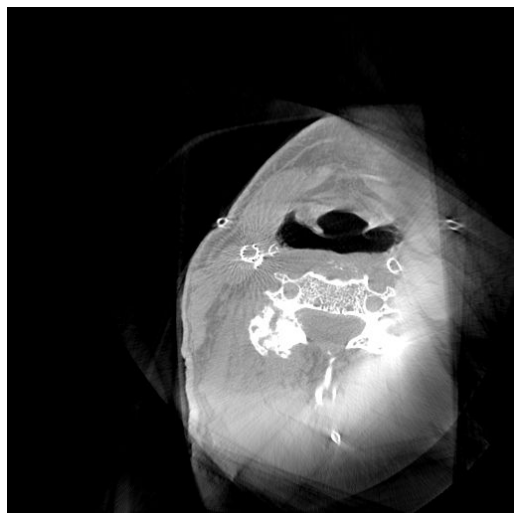

(b) Water cylinder correction, HU grayscale window $(-800,800)$.

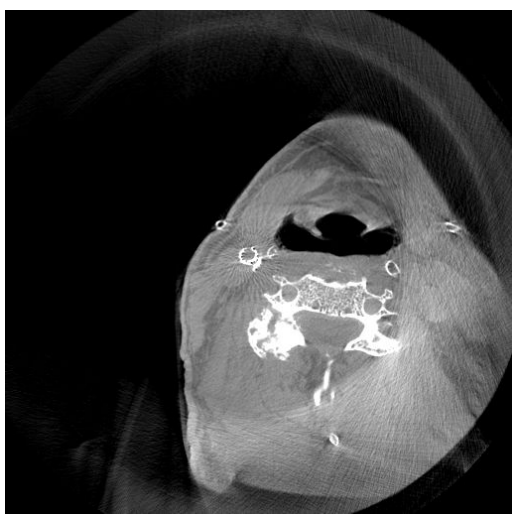

(c) Scattered radiation correction, HU grayscale window $(-800,800)$. (a) Projection image of the truncated dataset.
Figure 7: Projection image of the truncated dataset and the results of the water cylinder reconstruction and our new approach. The new approach was performed with the add+squared adjustment method and the SaS interpolation. Dataset 4.

\section{DISCUSSION}

A limitation of our method is that it cannot be applied if scattered radiation does not exist outside the FOV. This can however be detected and another truncation correction method can be applied, e.g. the water cylinder correction. We employ data outside the FOV which was actually measured and therefore is better able to represent the actual object. However, the adjustment of the data outside the FOV is purely heuristic. We expect even better results if the physical behavior of the scatter radiation is correctly modeled. Despite of these drawbacks, our new approach is, as presented, competitive with other methods. 


\section{SUMMARY}

In this paper, we presented a new method of truncation correction using scattered radiation. First tests have shown that the method works as well as other state-of-the-art correction methods, like the water-cylinder correction, which are already in clinical use. Our method has advantages if bones are truncated. Dense objects can be reconstructed in the area outside the FOV and the cupping effect is mostly not visible in our reconstruction results.

\section{ACKNOWLEDGMENT}

This work was supported by Siemens AG, Healthcare Sector. The reconstructions were done with a Siemens prototype software and not with a clinical product. The concepts and information in this paper were never submitted, published, or presented before. We thank Dr. Mawad at St. Luke's Medical Center in Houston for the permission to use his clinical data. The authors gratefully acknowledge funding of the Erlangen Graduate School in Advanced Optical Technologies (SAOT) by the German Research Foundation (DFG) in the framework of the German excellence initiative.

\section{REFERENCES}

[1] Chintalapani, G., Chinnadurai, P., Maier, A., Shaltoni, H., Morsi, H., and Mawad, M., "The Value of Volume of Interest (VOI) C-arm CT Imaging in the Endovascular Treatment of Intracranial Aneurysms A Feasibility Study," Proceedings of ASNR, 12-O-1509-ASNR (2012).

[2] Zeng, L., [Medical Image Reconstruction], Springer Verlag, Heidelberg, 1. ed. (2010).

[3] Kolditz, D., Kyriakou, Y., and Kalender, W., "Volume-of-interest (VOI) imaging in C-arm flat-detector CT for high image quality at reduced dose," Medical Physics 37(6), 2719-2730 (2010).

[4] Kudo, H., Courdurier, M., Noo, F., and Defrise, M., "Tiny a priori knowledge solves the interior problem in computed tomography," Physics in Medicine and Biology 53(9), 2207-2231 (2008).

[5] Hsieh, J., Chao, E., Thibault, J., Grekowicz, B., Horst, A., and McOlash, S., "A novel algorithm to extend the CT scan field-of-view," Medical Physics 31(9), 2385-2391 (2004).

[6] Maier, A., Scholz, B., and Dennerlein, F., "Optimization-based Extrapolation for Truncation Correction," 2nd CT Meeting, 390-394 (2012).

[7] Dennerlein, F. and Maier, A., "Region-of-interest reconstruction on medical c-arms with the ATRACT algorithm," Medical Imaging 2012: Physics of Medical Imaging 8313, p. 83131B, SPIE (2012).

[8] Xia, Y., Maier, A., Dennerlein, F., Hofmann, H., and Hornegger, J., "Efficient 2D Filtering for Cone-Beam VOI Reconstruction," IEEE MIC, 2415-2420 (2012).

[9] Lehmann, T., Oberschelp, W., Pelikan, E., and Repges, R., [Bildverarbeitung in der Medizin], Springer Verlag, Heidelberg (1997).

[10] Fischler, M. and Bolles, R., "Random sample consensus: A paradigm for model fitting with applications to image analysis and automated cartography," Communications of the ACM 24(6), 381-395 (1981).

[11] Schwemmer, C., Prümmer, M., Daum, V., and Hornegger, J., "High-Density Object Removal from Projection Images Using Low-Frequency-Based Object Masking," Proc. BVM , 365-369 (2010). 